\title{
Temporary Extrathoracic Vacuum Therapy Splint in Chest Wall Reconstruction
}

\author{
Srikanth Vasudevan ${ }^{1}$ Shriram Vaidya ${ }^{2}$ Ritu Baath $\mathrm{S}^{1} \quad$ Ashok Basur C $^{1}$ \\ Anantheswar Yellambalase $N^{1}$ Sudarshan Reddy Nagireddy ${ }^{1}$
}

${ }^{1}$ Department of Plastic and Reconstructive Surgery, Manipal Hospital, Bangalore, India

${ }^{2}$ Department of Critical Care Medicine, Manipal Hospital, Bangalore, India

Indian J Plast Surg 2021;54:211-214.

\begin{abstract}
Address for correspondence Ritu Baath S, MBBS, DrNB (Plastic Surgery), Department of Plastic and Reconstructive Surgery, Manipal Hospital, Bangalore 560017, India (e-mail: dr.ritubatth@gmail.com).
\end{abstract}

Abstract
Keywords
- chest wall
reconstruction
- negative pressure
wound therapy
- paradoxical respiration

\section{Introduction}

Full-thickness chest wall defects allure a plastic surgeon for covering the underlying vital organs to maintain a satisfactory cardiopulmonary function and respiratory mechanics. The choice of reconstruction depends on surgeon's preference and experience, cost and availability of prosthesis, morbidity, and future prognosis. Paradoxical respiration is a sequel of loss of chest wall rigidity in the resected area which gives rise to impaired breathing mechanics and hemodynamic instability. Application of negative pressure wound therapy (NPWT) to a semirigid reconstruction with paradoxical movement can act as a bridge to recovery by improving altered breathing mechanics and its sequelae.

published online June 15, 2021
DOI https://doi.org/ $10.1055 / \mathrm{s}-0041-1729502$ ISSN 0970-0358

\section{Case Report}

A 38-year-old man presented with a $21 \mathrm{~cm} \times 22 \mathrm{~cm} \times 26 \mathrm{~cm}$ chondrosarcoma involving the right second to fifth ribs with

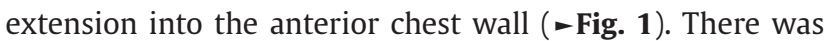
mild compressive atelectasis also. Wide local excision of the lesion led to a $24 \mathrm{~cm} \times 21 \mathrm{~cm}$ anterior chest wall defect with four missing ribs from the anterior axillary line to the midline and exposed cardiopulmonary structures.

Rib cage was reconstructed using a double-folded polypropylene mesh anchored using No. 1 prolene passed

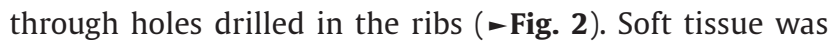
reconstructed using free anterolateral thigh flap (ALT). Nipple areola complex (NAC) was harvested as a free graft from the excised specimen and secured to the flap with a bolster dressing ( - Fig. 3 ). Patient was shifted to the intensive care

(C) 2021. Association of Plastic Surgeons of India.

This is an open access article published by Thieme under the terms of the Creative Commons Attribution-NonDerivative-NonCommercial-License, permitting copying and reproduction so long as the original work is given appropriate credit. Contents may not be used for commercial purposes, or adapted, remixed, transformed or built upon. (https://creativecommons.org/licenses/by-nc-nd/4.0/).

Thieme Medical and Scientific Publishers Pvt. Ltd. A-12, 2nd Floor, Sector 2, Noida-201301 UP, India 


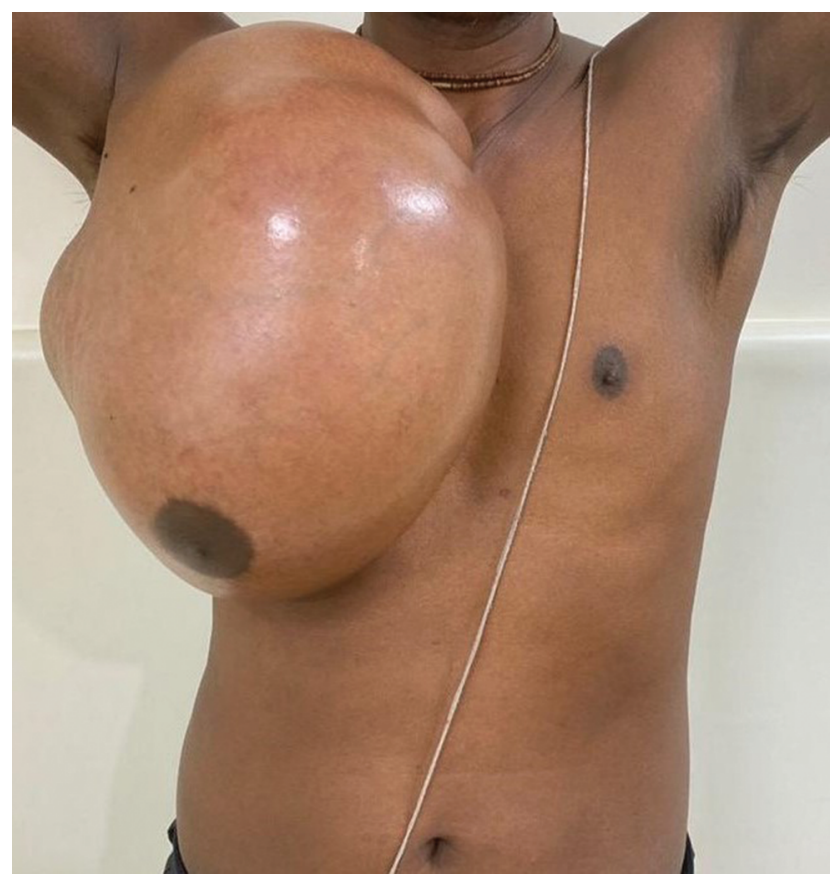

Fig. 1 Chest wall tumor-anteroposterior view.

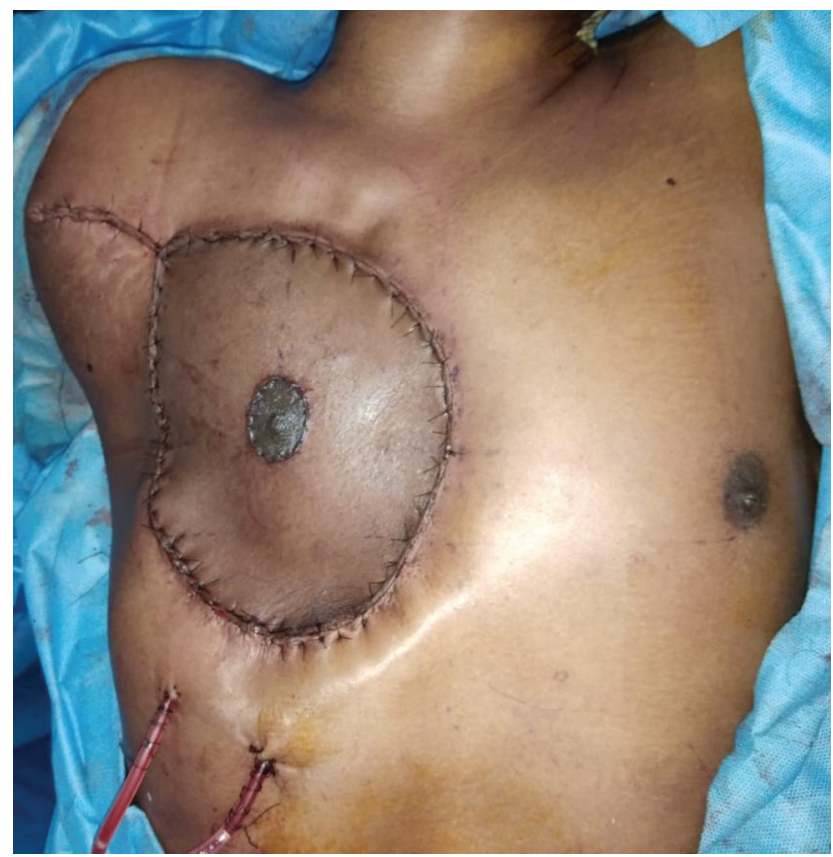

Fig. 3 Post flap inset and nipple areola complex (NAC) reconstruction with free NAC graft.

unit (ICU) with an endotracheal tube and $0.05 \mu \mathrm{g} / \mathrm{kg} / \mathrm{min}$ noradrenaline because of intraoperative hemodynamic instability. Injection vasopressin was added on postoperative day 2 as hemodynamic instability worsened during attempts at spontaneous breathing. Spontaneous breathing trials (SBT) and weaning from ventilator support were unsuccessful. On postoperative day (POD) 1, pneumothorax was detected beneath the flap for which a chest tube was inserted ( - Fig. 4). Echocardiogram revealed normal left ventricular ejection fraction. Antibiotics were escalated on

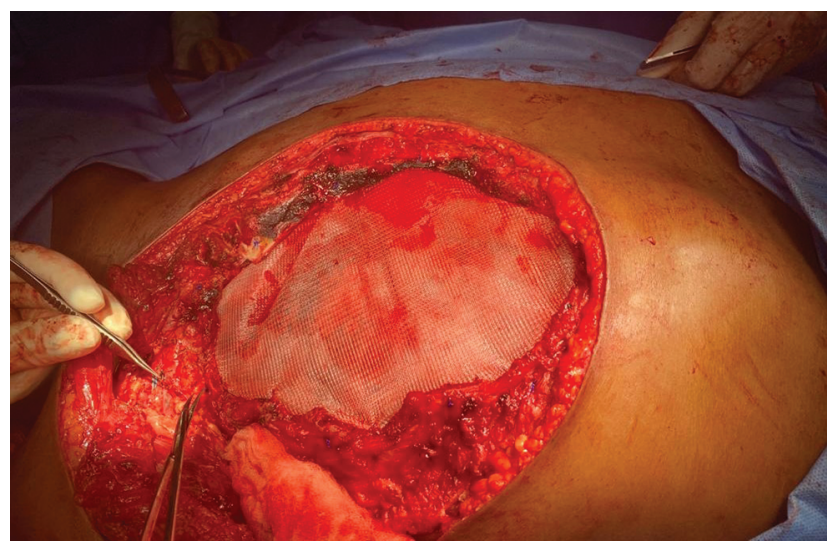

Fig. 2 Rib cage reconstruction with prolene mesh.

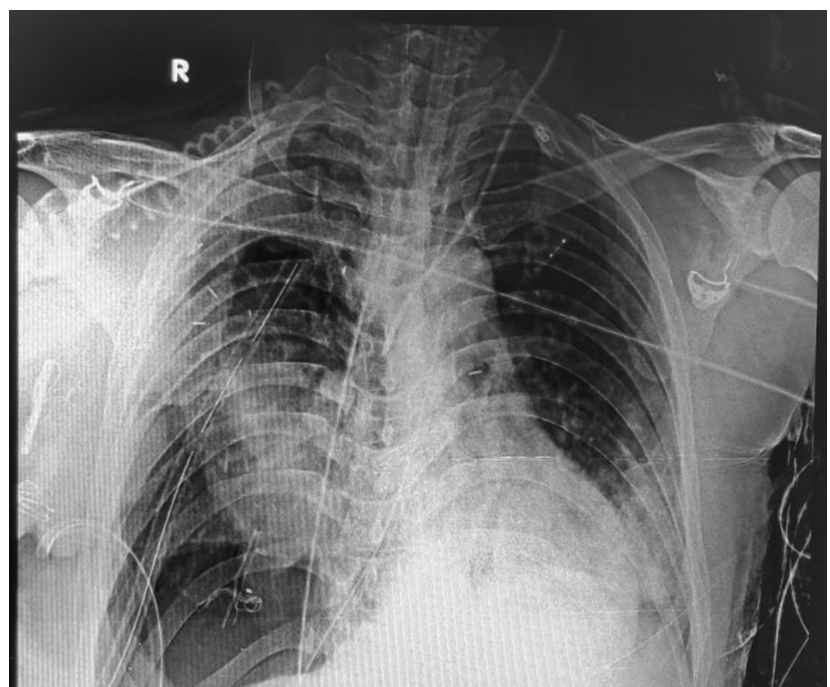

Fig. 4 Chest $X$-ray on postoperative (POD) 1 showing minimal pneumothorax under the flap.

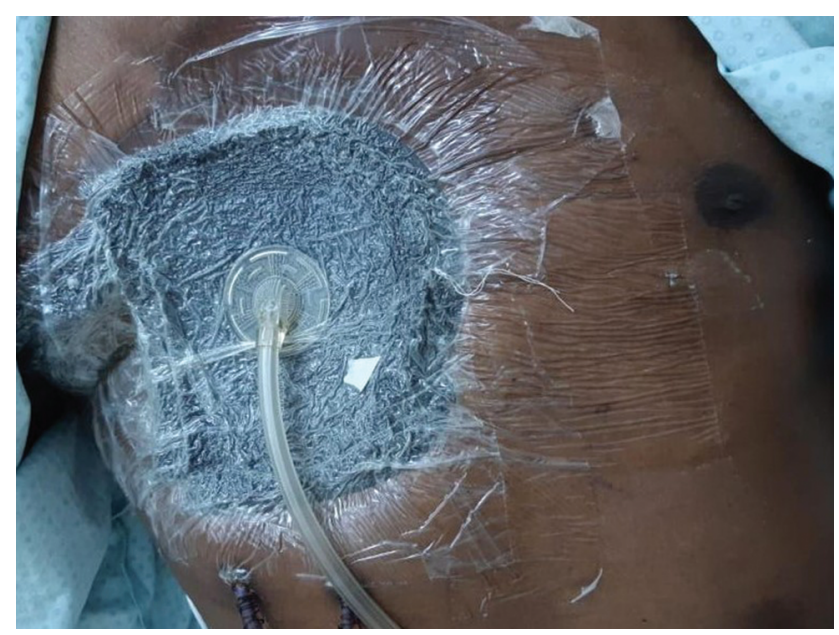

Fig. 5 Negative pressure wound therapy applied to chest.

day 2 but blood culture came negative and patient remained afebrile.

On POD 5, after relevant literature search, a vacuum dressing was applied over the flap using a polyurethane sponge and film at $-125 \mathrm{~mm} \mathrm{Hg}^{1}$ (-Fig. 5). The sponge extended 
$2 \mathrm{~cm}$ beyond the bony defect to avoid direct inward pressure. Consistent improvement was noticed in breathing mechanics over the next 3 days evidenced by reduced requirement of positive end expiratory pressure (PEEP) and better tolerance of SBT and pressure support ventilation (PSV) (- Table 1). The patient was extubated on POD 8, transitioned to high-flow nasal cannula, and shifted out of ICU on POD 10. Upon removal of vacuum dressing, the flap, suture line, and free NAC graft were healthy. The patient was discharged with NPWT and satisfactory lung expansion on POD 11 (-Fig. 6). On POD 15, NPWT was stopped and he had minimal residual paradoxical breathing, with no discomfort in breathing.

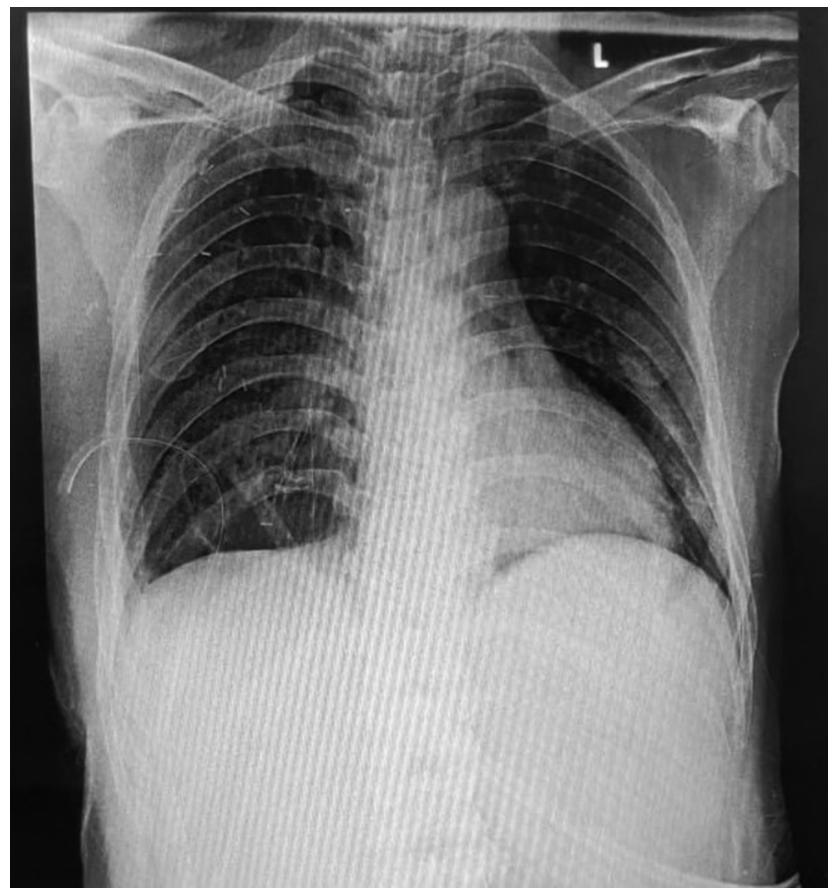

Fig. 6 Chest X-ray on discharge showing satisfactory lung expansion.

\section{Discussion}

Chest wall defects $>5 \mathrm{~cm}$ or involving $>4$ ribs demand reconstruction to avoid organ herniation and respiratory paradox. Reconstruction methods include synthetic, biological, or composite mesh; titanium plates or mesh; silicon tubes; bone cement; allografts; or prefabricated implants. ${ }^{2}$ Function of the chest wall is not limited to static support and protection, rather it plays a pivotal role in the mechanics of respiration. Comparatively, a double-folded prolene mesh lacks initial stability but subsequently attains rigidity by the process of fibrous ingrowth of surrounding tissue. Methods like sandwich technique with polymethyl methacrylate (PMMA) cement between two layers of prolene mesh can convert a semirigid mesh reconstruction into rigid but authors avoid this technique due to complications like exothermic reaction, skin burn, pain, fracture, infection, and metabolic acidosis., ${ }^{3,45}$ Among patients with prolonged incapability to attain satisfactory breathing mechanics during immediate postoperative period, a temporary external support can assist liberation from ventilation.

We also analyzed the possible causes for persistent vasopressor requirement like systemic inflammatory response syndrome (SIRS), septic shock, and cardiogenic shock. SIRS was unlikely as the requirement continued even on the fifth postoperative day. Septic shock was ruled out as there was no obvious source, blood cultures were negative, patient remained afebrile, and shock persisted even after escalation of antibiotic. Normal contractile function of heart on echocardiogram ruled out cardiogenic shock. There was temporal association between application of NPWT and resolution of shock.

Over the first four postoperative days, the patient could not tolerate attempts at weaning because of paradoxical movement of flap and desaturation. Even though he tolerated support ventilation with higher pressure support (PS) levels, attempts at reduction of PS led to episodes of desaturation; hence, intermittent controlled ventilation was continued. After application of NPWT, the patient could tolerate lower pressure support levels.

During spontaneous breathing, transpulmonary pressure difference $\left(\mathrm{P}_{T \mathrm{~T}}\right)$, that is, the difference between alveolar pressure $\left(\mathrm{P}_{\mathrm{A}}\right)$ and intrapleural pressure $\left(\mathrm{P}_{\mathrm{pl}}\right)$, drives the flow of air from airway to alveoli. This requires contraction of diaphragm and muscles in the chest wall to create negative $\mathrm{P}_{\mathrm{pl}}$. In this case, during inspiration when the patient is breathing spontaneously on lesser pressure support levels, ability of the right hemithorax to generate negative $\mathrm{P}_{\mathrm{pl}}$ is much lesser than the left hemithorax because of chest wall defect covered by semirigid flap which moves inwards during inspiration. This difference in $\mathrm{P}_{\mathrm{pl}}$ between two hemithoraces causes movement of air from right to left lung. During expiration, passive recoil of normal left chest wall is more efficient compared with right side, causing the movement of air from left lung to right lung, leading to ineffective ventilation and gas exchange abnormalities ( - Fig. 7 ). ${ }^{6}$

Table 1 Postoperative mode of ventilation and trend of oxygenation

\begin{tabular}{|c|c|c|c|c|c|c|c|c|}
\hline POD & 1 & 2 & 3 & 4 & 5 (VAC applied) & 6 & 7 & 8 \\
\hline $\mathrm{FiO}_{2}$ & 0.4 & 0.4 & 0.6 & 0.4 & 0.4 & 0.4 & 0.4 & 0.4 \\
\hline $\operatorname{PEEP}\left(\mathrm{cmH}_{2} \mathrm{O}\right)$ & 5 & 5 & 12 & 10 & 6 & 5 & 5 & \multirow{2}{*}{$\begin{array}{l}\text { Extubated to } \\
\text { HFNC }\end{array}$} \\
\hline $\mathrm{PaO}_{2}(\mathrm{~mm} \mathrm{Hg})$ & 86.4 & 69.0 & 70.1 & 94.2 & 63.7 & 58.6 & $V_{B G}$ & \\
\hline Mode of ventilation & \multicolumn{4}{|c|}{$\begin{array}{c}\text { VCV } \\
\text { (poor tolerance to PSV) }\end{array}$} & $\begin{array}{l}\text { PSV } \\
\text { (intermittent) }\end{array}$ & $\begin{array}{l}\text { PSV } \\
\text { (intermittent) }\end{array}$ & PSV & \\
\hline
\end{tabular}

Abbreviations: $\mathrm{FiO}_{2}$, fraction of inspired oxygen; $\mathrm{HFNC}$, high flow nasal cannula; $\mathrm{PaO}_{2}$, arterial partial pressure of oxygen; PEEP, positive end expiartory pressure; PSV, pressure support ventilation; VBG, venous blood gas analysis; VCV, volume-controlled ventilation.

${ }^{\mathrm{aPaO}}{ }_{2}$ cannot be assessed. 


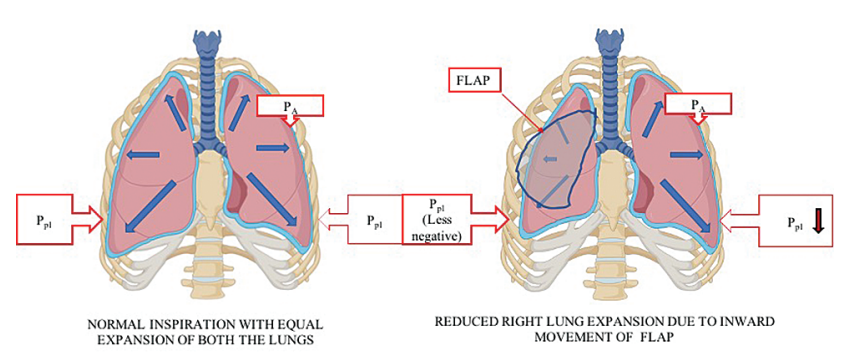

Fig. 7 Illustration showing the altered breathing mechanics in the patient postoperatively.

The fundamental equation of mechanical ventilation is $\mathrm{P}_{\text {mus }}=\mathrm{P}_{\text {res }}+\mathrm{P}_{\mathrm{el}}$, where $\mathrm{P}_{\text {mus }}$ is the pressure required to overcome the resistance $\left(\mathrm{P}_{\text {res }}\right)$ and elastance $\left(\mathrm{P}_{\mathrm{el}}\right)$ of lung using chest wall and diaphragm muscular function. Even a normal lung has a tendency to collapse inwards because of its natural elastance, which is resisted by surfactant and a negative intrapleural pressure generated by $\mathrm{P}_{\text {mus }}$, which needs an intact chest wall to generate it. Loss of significant portion of chest wall can lead to generation of less than adequate $P_{\text {mus }}$ which can be insufficient to overcome the natural elastance $\left(\mathrm{P}_{\mathrm{el}}\right)$ of lung even if the lung is healthy. This may have led to atelectasis of segment of lung underlying the flail flap. Application of NPWT to the flail flap could have contributed to temporary stabilization of right chest wall. The possible need of tracheostomy was also averted, unlike previous reports. ${ }^{1}$

NPWT acted as an interim external splint till fibrosis sets in. Although mature fibrous tissue formation takes longer than 15 days, NPWT would have aided the recovery by reducing atelectasis and mediastinal swing, cumulatively resulting in improved mechanics of breathing. The effect of NPWT on the flap microcirculation cannot be asserted by this report and it is still controversial. ${ }^{7}$ There have been studies that prove no negative impact of NPWT on microcirculation of flaps but rather show a positive impact of NPWT on flap edema and venous outflow; but these studies have suggested lack of sufficient evidence. ${ }^{8,9,10,11}$ International consensus dictates use of NPWT on free flaps with at lower negative pressures from -75 to $-100 \mathrm{~mm} \mathrm{Hg}$ and using implantable Doppler probes for monitoring. ${ }^{6}$ In this case no vascular complications were observed in the free tissue transferred. Hence the authors would like to reiterate the fact that using NPWT on a free flap should not be ruled out completely in lieu of probable vascular compromise.

\section{Conclusion}

External negative pressure splint could be considered as an early postoperative tool in patients with extensive chest wall reconstruction with semirigid techniques and postoperative paradoxical chest wall movement resulting in difficult weaning. It can decrease the need for elaborate hardware reconstruction and related complications by assisting recovery.

\section{Financial Disclosures}

None.

\section{Earlier Presentation}

None.

\section{Conflict of Interest}

None declared.

\section{Acknowledgments}

None.

\section{References}

1 Winge R, Berg JO, Albret R, Krag C. VAC for external fixation of flail chest. Clin Pract 2012;2(3):e65

2 Sanna S, Brandolini J, Pardolesi A, et al. Materials and techniques in chest wall reconstruction: a review. J Vis Surg 2017;3:95

3 Aghajanzadeh M, Alavy A, Taskindost M, Pourrasouly Z, Aghajanzadeh G, Massahnia S. Results of chest wall resection and reconstruction in 162 patients with benign and malignant chest wall disease. J Thorac Dis 2010;2(2):81-85

4 McCormack PM. Use of prosthetic materials in chest-wall reconstruction. Assets and liabilities. Surg Clin North Am 1989;69(5):965-976

5 Novoa N, Alcaide J, Gomez Hernández M, Fuentes M, Goñi E, Jimenez Lopez M. Chest wall-reconstruction: yesterday, today and the future. Shanghai Chest 2019;3:1-9

6 Harada K, Saoyama N, Izumi K, Hamaguchi N, Sasaki M, Inoue K. Experimental pendulum air in the flail chest. Jpn J Surg 1983;13(3):219-226

7 Krug E, Berg L, LeeC, et al. International Expert Panel on Negative Pressure Wound Therapy [NPWT-EP]. Evidence-based recommendations for the use of negative pressure wound therapy in traumatic wounds and reconstructive surgery: steps towards an international consensus. Injury 2011;42(Suppl 1) :S1-S12

8 Vaienti L, Gazzola R, Benanti E, et al. Failure by congestion of pedicled and free flaps for reconstruction of lower limbs after trauma: the role of negative-pressure wound therapy. J Orthop Traumatol 2013;14(3):213-217

9 Chim H, Zoghbi Y, Nugent AG, Kassira W, Askari M, Salgado CJ. Immediate application of vacuum assisted closure dressing over free muscle flaps in the lower extremity does not compromise flap survival and results in decreased flap thickness. Arch Plast Surg 2018;45(1):45-50

10 Yu P, Yu N, Yang X, Jin X, Lu H, Qi Z. Clinical efficacy and safety of negative-pressure wound therapy on flaps: a systematic review. J Reconstr Microsurg 2017;33(5):358-366

11 Opoku-Agyeman JL, Matera DV, Simone JE, Behnam AB. Flap viability after direct immediate application of negative pressure wound therapy on free flaps: a systematic review and pooled analysis of reported outcomes. Journal of Reconstructive Microsurgery Open 2019;04(2):e77-e82 